\title{
PATRONES DIFERENCIALES EN LA ELABORACIÓN DE RESULTADOS EN BEBÉS TÍPICOS Y BEBÉS CON SÍNDROME DE DOWN $(\mathbf{1 ; 3} \text { a. })^{1}$
}

\author{
Sylvia Sastre i Riba \\ Elena Escolano Pérez \\ Universidad de La Rioja
}

\begin{abstract}
RESUMEN: Este trabajo pretende, desde una perspectiva neuroconstructivista del desarrollo cognitivo, conocer y comparar la organización de la actividad lógica en la elaboración de un resultado en sujetos con cursos de desarrollo diferencial: bebés típicos y bebés con Síndrome de Down, en un momento de desarrollo cognitivo equivalente a 1;3 años. Aplicando la metodología observacional se estudian 10 bebés: cinco bebés típicos y cinco bebés con Síndrome de Down. Se registra y codifica la actividad espontánea de cada bebé ante una tarea abierta no verbal que posibilita la elaboración de resultados de distinta complejidad. El análisis secuencial, progresivo y retrospectivo, permite conocer el curso de acción en cada grupo de bebés para elaborar un resultado. A partir de los patrones conductuales intragrupo, se establecen las diferencias intergrupales en dicho proceso. Los resultados muestran un patrón de elaboración de resultados más complejo y flexible entre los bebés típicos que entre los bebés con Síndrome de Down.
\end{abstract}

ABSTRACT: The aim of this study is to get to know and compare the logical action organization in the elaboration of results among babies with alternative developmental courses (typical babies and Down's Syndrome ones with a cognitive developmental level of $1 ; 3$ years) from a neuroconstructivist point of view based on infant cognitive development. Using systematic observation, we studied ten babies: five typical babies and five Down's Syndrome ones. We recorded and coded the spontaneous activity of the babies in a non-verbal behaviour-oriented task that allowed them to elaborate results of different complexity. The sequential analyses, both progressive and retrospective, enabled us to identify the course of action to elaborate results in each group

1. Esta investigación ha recibido el soporte del Proyecto Nacional de Investigación PB980207-C02-01, así como de la beca F.P.U. del M.E.C. (AP2001-0553). 
of babies. From the intra-group behavioural patterns, we established intergroup differences in the process. The results showed a pattern of elaboration of results more complex and flexible in typical babies compared to Down's Syndrome ones.

PALABRAS CLAVE: Desarrollo cognitivo, organización lógica de la acción, elaboración de resultados, bebés típicos, bebés con Síndrome de Down.

KEYWORDS: cognitive development, logical action organization, elaboration of results, typical babies, Down's Syndrome babies.

\section{UNA APROXIMACIÓN AL DESARROLLO TEMPRANO}

La perspectiva neuroconstructivista del desarrollo cognitivo (Mareschal \& Schultz, 1997; Quartz \& Sejnowski, 1994, 1997), defiende que éste es el resultado de la interacción entre la configuración estructural del cerebro y su regulación a través de la actividad con el medio, destacando la importancia del bebé como sujeto activo en el desarrollo. Los procesos de maduración cerebral, condicionados por el contenido del código genético, posibilitan la aparición de ciertas competencias que, mediante la experiencia del sujeto y la estimulación que recibe del medio, permitirán progresivamente la transformación de las estructuras iniciales y la emergencia y desarrollo de otras. Este proceso es especialmente importante durante los tres primeros años de vida y, entre ellos, en particular, en el intervalo comprendido entre el nacimiento y los 18 primeros meses de vida.

Cada vez son más numerosas las investigaciones que, desde esta perspectiva, ofrecen datos empíricos sobre las relaciones existentes entre desarrollo cognitivo y la estructura neurobiológica, tratando incluso de llegar a establecer los correlatos neurológicos de distintas funciones cognitivas. Se ha demostrado, por ejemplo, que durante el primer año de vida extrauterina se producen importantes cambios madurativos en la estructura y funcionamiento del lóbulo frontal, y dentro de él en el córtex prefrontal, relacionados con la progresiva mielinización, crecimiento dendrítico y celular, establecimiento de nuevas rutas sinápticas y activación de sistemas neuroquímicos (Benes, Taylor \& Cunningham, 2000; Christoff \& Gabrieli, 2000; Stuss \& Knight, 2002). Estos cambios coinciden con importantes ganancias en la competencia cognitiva del bebé durante ese período, así como con la puesta en funcionamiento de las funciones ejecutivas (Diamond, 2001, 2002), entendiendo éstas, como un conjunto de procesos cognitivos de alto nivel cuya característica común es la coordinación del procesamiento de la información y el control de la conducta con el propósito de lograr objetivos tanto a corto como a largo plazo (Heyder, Suchan \& Daum, 2004; Zelazo, Craik \& Booth, 2004).

Entre las diversas funciones ejecutivas se encuentran, por ejemplo, la capacidad de organizar y planificar una tarea, seleccionar apropiadamente los objetivos, iniciar y mantener un plan mientras se ejecuta, inhibir las distracciones o interferencias, cambiar de estrategias de modo flexible si el caso lo requiere, autorregular y controlar el curso de la acción para asegurar que la meta propuesta puede conseguirse, etc. Todas ellas, son requisitos para la resolución de un problema, puesto que per- 
miten la optimización de la ejecución en las tareas dirigidas hacia una meta u objetivo (Anderson, Levin \& Jacobs, 2002).

La consecución de estos objetivos intencionales es lo que posibilita y dirige la acción. "Los humanos Ilevan a cabo acciones con el fin de alcanzar objetivos, es decir, con el fin de crear o modificar algún evento o estado de cosas de acuerdo con sus intenciones" (Hommel, 2003, p. 593). El resultado obtenido "es el hilo conductor del desarrollo del curso de la acción" (Pastor y Sastre, 1994, p. 200).

Tanto los adultos como los niños organizan su memoria sobre los hechos en torno a estos objetivos de acción (Zacks \& Tversky, 2001) que sirven como constructos explicativos y claves predictivas para describirla. Así pues, el objetivo o resultado de la acción sirven como un armazón organizacional que juega un poderoso papel como mediador del aprendizaje.

La capacidad para detectar la estructura del objetivo de la actividad emerge durante la infancia, alcanzando mayor complejidad conforme avanza la maduración cognitiva. Durante el primer año de vida, los niños son capaces de interpretar las acciones humanas (Sommerville \& Woodward, 2005; Woodward \& Guajardo, 2002; Woodward \& Sommerville, 2000; Woodward, Sommerville \& Guajardo, 2001) y el movimiento de ciertos objetos (Csibra, Biro, Koos \& Gergely, 2003) en base a los resultados hacia los que se dirigen, más que en términos de sus características perceptivas superficiales. Por ejemplo, tras observar que un actor coge repetidamente un juguete, los bebés muestran mayor respuesta de novedad ante el cambio del objetivo previsto (es decir, cuando no coge el juguete) que ante el cambio de la localización espacial de éste o de la trayectoria de prensión (Woodward, 1998).

A pesar de la intencionalidad de la acción, evidente durante el primer año de vida, los objetivos o metas son cambiantes, mostrando la dificultad del bebé para mantenerlos, inhibiendo otros. La consecución del mantenimiento de un objetivo se logra de forma estable en torno a los tres años de edad.

Así pues, durante la infancia, y muy especialmente entre el año y los tres años de edad, la capacidad para organizar la actividad en torno a un objetivo sostenido aumenta rápidamente (Jennings, 2004), paralelamente al desarrollo y perfeccionamiento de las funciones ejecutivas y otras capacidades cognitivas que permiten la inhibición de una respuesta preponderante y la selección de esquemas pertinentes, la representación mental (Bruner, 1984; Mandler, 1998; Mounoud, 1983), la organización lógica de la acción (Langer, 1980, 1986, 2000) y la construcción del saber, habilidades todas ellas requeridas para mantener una estrategia de solución de problemas hacia la consecución de un objetivo.

Entre estas capacidades cognitivas, destaca la lógica, entendiéndola como la capacidad mediante la cual el sujeto puede "captar información significativa, organizarla y operar sobre el medio, obteniendo como resultado el conocimiento", tanto físico como lógico-matemático (Pastor y Sastre, 1994, p. 202).

Langer (1980) postula que, desde el inicio de la interacción del bebé con el medio, hay una forma elemental de lógica que se construye a través de la acción organizada y significativa (lógica en acción o protológica en términos de Langer (1986)), que más tarde se interioriza y se convierte en una lógica de enunciados ligada a códigos simbólicos. Por tanto, toda operación lógica tiene su origen en la actividad organizada del 
niño durante el primer año de vida. La acción constituye el medio a través del cual el bebé consigue la información necesaria para construir la organización lógica del mundo físico y social que le rodea, así como su posterior representación. Consecuentemente, la acción es una herramienta básica en la construcción intelectual, siempre que esté organizada (Langer, 1986; Pastor y Sastre, 1994).

Por lo tanto, de acuerdo con Langer (1990), conocer implica actuar pero no toda acción produce conocimiento. Es decir, la acción organizada sobre el entorno permite la sucesiva elaboración del conocimiento, tanto del conocimiento físico (cuando el bebé centra su atención sobre las propiedades de los objetos) como del conocimiento lógico-matemático (focalización de la atención en las acciones realizadas sobre ellos). Para ello, el bebé aplica los elementos que componen la lógica: las funciones y las operaciones, respectivamente (Langer, 1980).

Las funciones se refieren a los cambios o transformaciones que provocan las acciones sobre los objetos mediante la exploración y experimentación de sus propiedades físicas, obteniendo informaciones respecto a cómo éstos responden a estas acciones (conocimiento físico) (Bideaud, 1988; Langer, 1982; Piaget, 1976, 1983). Existen tres tipos de funciones:

* Medio-fin: comprende las relaciones de dependencia física entre los objetos para lograr una finalidad o efecto (Pastor y Sastre, 1994). Ejemplo: utilizar una vara o bastón (medio) para acercar un objeto que no está al alcance de la mano y poder así cogerlo (fin).

* Causa-efecto: comprende la relación entre una acción y el resultado consecuente. Ejemplo: empujar un objeto con otro.

* Organización espacial: supone la ordenación y distribución espacial de distintos objetos realizando alineamientos horizontales o verticales, relaciones de proximidad-lejanía, etc. (Pastor y Sastre, 1994).

Las operaciones no se refieren a los objetos concretos y sus propiedades físicas sino a las acciones realizadas y las relaciones establecidas sobre ellos (Adición, Sustracción, etc). Se trata, por lo tanto, de una reflexión del sujeto acerca de la organización y coherencia de sus propias acciones, su producto es el conocimiento lógico-matemático. De entre los tres tipos de operaciones establecidas (de Combinación, de Relación y Condicionales), destacan (Langer, 1986; Pastor y Sastre, 1994):

1) Operaciones de Combinación: comprenden las operaciones de Composición, Descomposición, Deformación y Recomposición. La actividad se dirige hacia la construcción de un producto estable (elementos, colecciones) por agrupación de objetos según similitudes y diferencias.

2) Operaciones de Relación: comprenden las operaciones de Adición, Sustracción, Multiplicación y División. Estas operaciones se aplican a los productos de las operaciones de Combinación (elementos y colecciones), generando relaciones cuantitativas entre ellos. El interés no radica en la estabilidad del producto final (como sucede en las operaciones de Combinación), sino en la acción que se está llevando a cabo. 
Por lo tanto, el desarrollo de la protológica (lógica en acción de las primeras edades) desempeña un papel especialmente relevante en el desarrollo cognitivo del sujeto desde su inicio.

No obstante, a pesar de esta trascendencia, son escasos los trabajos sobre su estudio, más aún desde un abordaje diferencial que considere poblaciones infantiles con factores de riesgo o con patologías establecidas desde el nacimiento, pese a los índices de prevalencia e incidencia que alcanzan algunas de estas poblaciones durante los últimos años (Poch, Sastre, Merino, Escolano, Farriol y Gil, 2003).

De acuerdo con lo expuesto, este trabajo pretende, comparativa y diferencialmente, conocer la organización lógica de la actividad en la obtención de un resultado entre sujetos con cursos de desarrollo alternativo: bebés típicos (BT) y bebés con Síndrome de Down (BD) a la edad de 1;3 años. En referencia a ello, cabría esperar un proceso de obtención de resultados más complejo y flexible entre los bebés típicos que entre los bebés con Síndrome de Down.

\section{MÉTOdo}

\section{Participantes}

La muestra de estudio está compuesta por cinco bebés típicos y cinco bebés con Síndrome de Down. Los primeros han sido estudiados a una edad cronológica de $1 ; 3$ años y los segundos a una edad de desarrollo cognitivo equivalente, de acuerdo con los resultados de su evaluación en la Escala de Observación Sistemática de 0 a 3 años de Cambrodí y Sastre (1993), independientemente de su edad cronológica, no superior a 2;6 años.

La extracción de la muestra ha seguido un procedimiento no probabilístico en función de los objetivos determinados previamente. Para acceder a los bebés típicos se contactó con una escuela infantil de Logroño. La muestra de bebés con Síndrome de Down se extrajo a través del Equipo de Pediatría del Hospital San Millán de Logroño y de la Fundación Hospital de Calahorra. En ambos casos se presentó a los padres la investigación y se les solicitó consentimiento por escrito.

\section{Material}

El material de estímulo consiste en una tarea no verbal abierta que permite la ejecución de distintos cursos de acción lógica organizada (ver Figura 1). Está compuesto por:

1. Un cajón contenedor de madera, fabricado "ad hoc" $(60 \times 25 \times 20 \mathrm{~cm}$.) en cuya superficie se distinguen tres zonas con características físicas y funcionales diferentes:
a) una zona blanca imantada,
b) una zona azul donde hay insertado un cubilete amarillo con una tapa con tres orificios que se corresponden con las tres formas de las piezas (redon- da, cuadrada y triangular),
c) una ranura horizontal de $4 \mathrm{~cm}$. de ancho a lo largo de todo un lateral de la caja de madera. 
2. Doce figuras geométricas de plástico con un pequeño imán circular en una base, de color diferente en función de su forma: cuatro prismas de color verde, cuatro cubos rojos y cuatro cilindros azules.



Figura 1. Material

\section{Procedimiento}

El bebé está sentado en el suelo frente al material de estímulo y junto a un adulto que actúa bajo la consigna de no intervenir directamente sino, sólo, a partir de: a) una demanda infantil, b) una actividad repetitiva o c) cuando ésta se paralice. Otro adulto está presente en la sala sin intervenir y alejado del bebé, a cargo de la cámara de registro. Se ha eliminado cualquier posible estímulo distractor en la sala: juguetes a la vista del niño, presencia de otros compañeros, etc.

El material se presenta con el continente tapado y con todas las piezas en su interior. En los casos en los que el bebé no inicia una acción, el adulto motiva a ello, despertando su interés hacia los objetos.

Cada participante ha sido registrado durante una sesión de 15 minutos de duración media. Los bebés típicos han sido registrados en la escuela infantil a la que asisten; los bebés con Síndrome de Down en el Hospital San Millán, al que acuden con regularidad.

La conducta de los bebés, se analiza microgenéticamente. Este análisis tiene tres niveles:

* Primer nivel: Transcripción y codificación de la realidad, sujeto a sujeto. Supone el vaciado de los datos registrados en formato VHS a formato digital y pos- 
teriormente a formato papel (hojas de transcripción o de vaciado de datos empíricos creadas ad hoc) mediante su descripción exhaustiva y ordinal, constituyendo la base de todos los análisis posteriores de las acciones.

* Segundo nivel: Incluye: a) la adaptación del "Sistema de registro observacional compuesto adaptado" (SROCA) (ver Escolano, 2003; Sastre, Escolano y Merino, 2004); b) el control de la calidad del dato, y c) la aplicación definitiva del SROCA. En concreto:

a. Adaptación del "Sistema de registro observacional compuesto adaptado" (SROCA) a partir de otros sistemas de análisis existentes (Pastor y Sastre, 1994; Sastre et al., 2000; Villares, 1999).

b. Control de la calidad del dato. Para ello, el SROCA se aplica al registro sistematizado de algunos participantes (y no de todos ellos), extraídos aleatoriamente mediante el programa informático "Aleatori" (Vargas, 1999). Las unidades de observación así seleccionadas, son codificadas mediante el SROCA en tres momentos temporales distantes entre sí en 15 días. Para cada una de ellas, se calcula el control de la calidad del dato intraobservador mediante el cálculo del Coeficiente $\alpha$ de Krippendorf (1980) en su forma canónica. En todos los casos se obtienen valores satisfactorios que revelan la consistencia intraobservador, y por tanto, aseguran la calidad del dato.

c. Aplicación definitiva del SROCA al registro sistematizado de cada uno de los participantes tras conocer los coeficientes de calidad del dato, codificando las unidades de conducta que dotan de contenido y significado al curso de la actividad de resolución de todos los bebés.

* Tercer nivel: Está centrado en el proceso de elaboración de resultados, tanto a nivel intragrupal como intergrupal. Es decir: a) la extracción del patrón de conducta de cada grupo de bebés en el proceso de elaboración de resultados, y b) el establecimiento de las diferencias intergrupo en dicho proceso.

a. Los patrones de conducta intragrupal se obtienen aplicando el análisis secuencial, progresivo y retrospectivo, al curso de la actividad y las unidades de conducta que lo componen. Para ello, los datos de cada participante categorizados previamente mediante el SROCA se compilan en función del grupo al que pertenece el bebé (BT o BD), y se aplica a la conducta intragrupal el análisis secuencial de retardos paso a paso (o progresivo), partiendo de la categoría Resultado (RE) y, retrospectivamente.

De esta manera se obtienen los patrones o "conductas que se suceden con mayor cohesión que el mero azar" (Anguera, 1990, p. 202) en la elaboración del resultado, comparativamente (BT y BD).

El análisis secuencial se ha realizado con el programa informático GSW 4.1 (Bakeman y Quera, 2002).

b. Establecimiento de las diferencias intergrupo. Los patrones intragrupo obtenidos permiten el establecimiento de las diferencias intergrupales mediante la comparación entre ellos, teniendo en cuenta: a) el número de componentes (unidades de conducta) de cada patrón; b) tipología de estos componentes, y c) su organización. 
Con ello puede deducirse la mayor o menor complejidad y flexibilidad en el proceso de búsqueda y construcción de resultados entre los bebés típicos y los bebés con Síndrome de Down estudiados.

\section{ResultadOS}

De acuerdo con lo anteriormente expuesto, en primer lugar se presentan los patrones de resolución intragrupo (BT y BD) correspondientes a cada uno de los grupos estudiados, y posteriormente, se exponen las diferencias intergrupales halladas en dicho proceso.

\section{Patrones de elaboración de resultados intragrupo: BT y BD}

\subsection{Bebés típicos}

El patrón propio entre los BT para la obtención de resultados se caracteriza por:

a) Los BT obtienen distintos resultados que implican en su totalidad la co-ocurrencia de operaciones lógicas, tanto de Combinación (Composición (C), Descomposición (D) y Recomposición (RC)) como de Relación (Adición (A) y Sustracción (S)), es decir, muestran complejidad cognitiva. Consecuentemente, la actividad de los BT se centra en establecer relaciones de tipo lógicomatemático entre los objetos, no en las propiedades físicas de éstos, dirigiendo su acción e interés tanto hacia la construcción de productos estables (operaciones de Combinación) como a la propia acción que están llevando a cabo (operaciones de Relación). Estas operaciones lógicas que desembocan en el logro de un resultado, son el objetivo que guía y dirige la actividad, contribuyendo a la elaboración de resultados de carácter lógico-matemático.

b) Estos resultados tienen diferentes patrones de consecución en función de que el bebé haya aplicado operaciones de Combinación (C, D y RC), o bien operaciones de Relación (A y S).

Tal como se observa en la Figura 2, los resultados obtenidos mediante operaciones de Combinación (C, D y RC), originan patrones de estructura arbórea, variados y flexibles, con circuitos abiertos que presentan diferentes posibilidades de actividad, las cuales van siendo dotadas por los propios bebés de una progresiva organización y secuencialización, así como de un sentido y contenido lógico-matemático apropiado al tipo de resultado que atienden, con la consideración de la información que les aportan los resultados "parciales" obtenidos en el curso de la actividad. Las "rutas" constituidas en el curso de la actividad se van aglutinando y condensando a medida que se acercan a la consecución del Resultado, seleccionando las posibilidades de acción inicialmente realizadas, y emergiendo unidades de conducta más pertinentes para el resultado.

Por otro lado, los patrones en los que se aplican operaciones de Relación (A y S), son patrones lineales, formados exclusivamente por la sucesión alternativa de estos dos tipos de operación (Adición y Sustracción), concordantemente con el resultado, sin producto estable, sino centrado en la propia acción de añadir o sustraer elementos del material. 


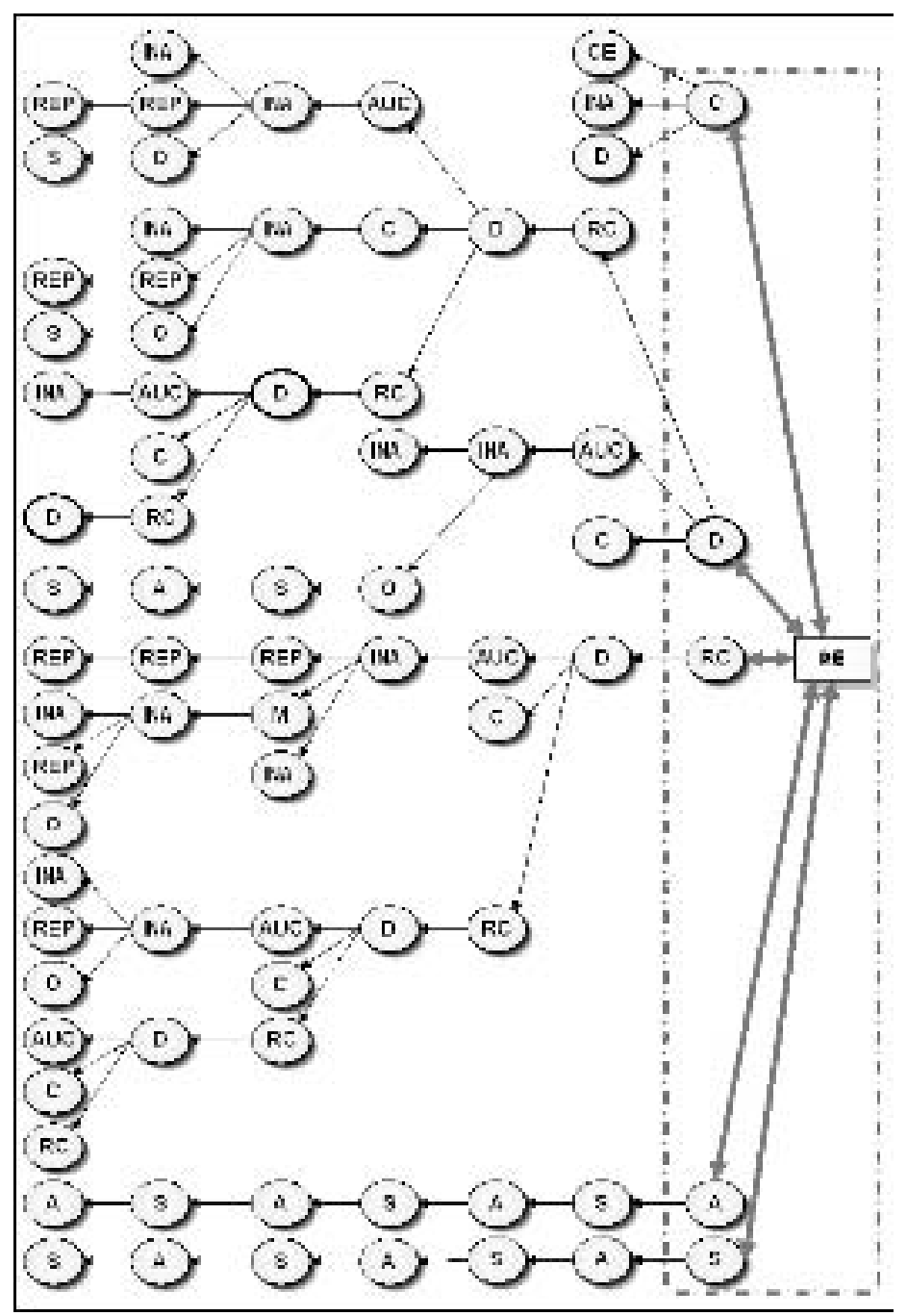

Figura 2. Proceso de construcción de resultados. Bebes Típicos

Dónde: $C$ = Operación de Combinación: Composición.

REP = Reproduce acción del adulto.

$D=$ Operación de Combinación: Descomposición.

INA = Intenta

$R C=$ Operación de Combinación: Recomposición.

$A \cup C=$ Autocorrige.

A = Operación de Relación: Adición.

$R E=$ Resultado.

S = Operación de Relación: Sustracción.

4- = Conductas secuenciales.

$O=$ Función: Organización espacial.

- Conductas co-ocurrentes.

M = Función: Medio-fin .

$C=$ Función: Causa-efecto.

$r ;$ = Zona obtención de resultados. 
c) La mayor parte de patrones desarrollados por los BT surgen por iniciativa propia de los bebés. El adulto apenas participa en la actividad, limitándose, cuando lo hace, a una propuesta de acción al inicio de la actividad, y nunca a lo largo de su desarrollo, siendo dicha propuesta reproducida por el bebé (REP) e insertada en su acción. De este modo, son los bebés los que inician, organizan y llevan a cabo la actividad dirigida hacia el logro de sus objetivos.

d) Se infiere la flexibilidad e intencionalidad subyacente a la acción de los bebés, dirigida persistentemente hacia el logro del resultado, siendo capaces de modular y monitorizar la acción hacia la obtención de un resultado deseado. Aunque, en algunos casos, al inicio de un curso de acción el bebé no consigue el éxito esperado (presencia de la categoría Intenta (INA) al inicio del $26,6 \%$ de las "rutas"), son capaces de detectar los elementos de error y autocorregir su acción (presencia a lo largo del patrón de la acción Autocorrige, AUC), de modo que no abandonan la actividad hasta alcanzar un resultado.

En resumen, el patrón de los BT destaca por la amplitud y riqueza en cuanto a cantidad de circuitos generados así como por su longitud y contenido lógico; siendo evidente la secuencialidad y dependencia de las diferentes acciones entre sí, dirigidas y organizadas hacia el logro de diferentes y abundantes tipos de resultados.

Asimismo, es de subrayar el control de los bebés sobre su propio curso de acción, en cuanto que son ellos quienes lo inician y desarrollan organizadamente, detectando y corrigiendo sus propios errores y, todo ello, de manera congruente con los resultados obtenidos.

\subsection{Bebés con Síndrome de Down}

El patrón conductual de los BD se representa en la Figura 3, caracterizado por los siguientes aspectos:

a) El curso de actividad es corto y sencillo, con cuatro "rutas" o circuitos de acción muy breves; lo que indica el escaso grado de relación existente entre las acciones desarrolladas. Se trata de acciones aisladas sin ningún fin que las dirija y organice.

b) Hay escasa iniciativa y gestión de la tarea, limitándose a la reproducción de la propuesta del adulto (REP), sin apenas enriquecerla con elementos propios.

c) El contenido lógico de la acción es pobre, las acciones que los bebés ejecutan por iniciativa propia (Composición (C) y Descomposición (D)), son los dos tipos de operaciones más sencillas, a nivel de desarrollo.

Todo ello evidencia un curso de acción pobre y corto, con un escaso número de acciones, siendo las existentes poco secuencializadas. 


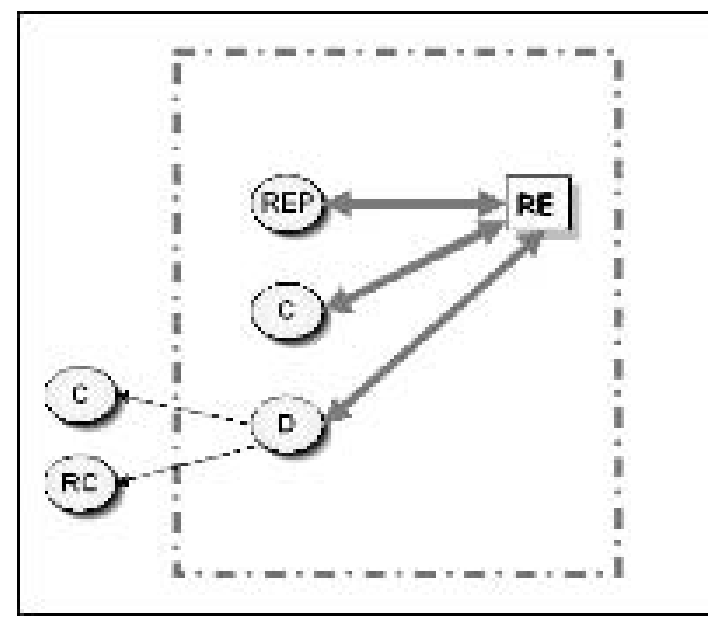

Figura 3. Proceso de construcción de resultados. Bebes con Síndrome de Down

Dónde: $\quad C=$ Operación de Combinación: Composición .

$R C$ = Operación de Combinación: Recomposición.

$D=$ Operación de Combinación: Descomposición.

$R E P=$ Reproduce acción del adulto.

$R E=$ Resultado.

$\downarrow-$ Conductas secuenciales.

- Conductas co-ocurrentes.

$r_{i}$ ! : Zona de obtención de resultados.

\section{Diferencias intergrupo: bebés típicos - bebés con Síndrome de Down}

Los BD obtienen resultados con su acción, aunque en menor medida que los BT, siendo de caráter "físico" más que "lógico-matemático".

Los patrones de los BT y los de los BD en su obtención presentan diferencias en los siguientes aspectos:

a) La secuencialidad de la acción, indicada a través de la longitud del patrón, es mayor en los $\mathrm{BT}$ que en los $\mathrm{BD}$, ejecutando los primeros acciones interrelacionadas frente a las acciones aisladas de los BD.

b) Las posibilidades de acción o circuitos de acción alternativos que se despliegan tras la ejecución de una determinada acción, muestran la flexibilidad/rigidez de la conducta y originan un patrón de estructura arbórea o lineal.

En este sentido, los BT desarrollan una acción flexible, con una importante riqueza y variedad de posibilidades de acción, todas ellas relacionadas, dirigidas y organizadas hacia un resultado. Esta actividad origina una extensa estructura arbórea o red de caminos posibles a seguir hasta el logro de un resultado.

Entre los BD, la secuencia de acción implica un circuito rígido y poco flexible, en el que tras la ejecución de una acción, no se abren distintas alternati- 
vas a seguir para la construcción de un resultado. La acción es única, determinando así una ruta de estructura lineal.

c) La complejidad del contenido del curso de la acción se refleja en las operaciones y funciones lógicas realizadas por cada grupo de bebés, así como en el resto de conductas asociadas a ellas.

Los BT ejecutan secuencias de acción con un contenido lógico rico y complejo. Estas secuencias están compuestas por pluralidad de acciones relacionadas entre sí y con el resultado. Por el contrario, los BD desarrollan un curso de acción más pobre, menos variado, sin un contenido cognitivamente tan complejo como el de los BT.

d) En cuanto a la gestión de la acción, los BT son protagonistas de la actividad que ejecutan, sin apenas participación del adulto, que interviene sólo puntualmente al inicio de la acción. Por lo tanto, los BT gestionan el variado y complejo contenido de su acción, siendo incluso capaces de detectar sus propios errores y autocorregirlos para el logro del resultado. Por otra parte, los BD muestran menor iniciativa y gestión, con una intervención más frecuente y directa del adulto que lo convierte en el gestor de la actividad.

e) La variedad y complejidad de las acciones co-ocurrentes a la obtención de los resultados es distinta.

Los BT obtienen variados y abundantes resultados ejecutando, por iniciativa propia, diversas operaciones lógicas tanto de Combinación como de Relación, estas últimas de mayor elaboración cognitiva. Sin embargo, los BD obtienen resultados: i) bien a partir de dos tipos de operaciones lógicas de Combinación: Composición (C) y Descomposición (D), (que además constituyen las operaciones más elementales); ii) o bien a partir de reproducciones de acciones propuestas por el adulto (REP), obteniendo por tanto menor variedad de resultados y además de menor complejidad.

En resumen, y de acuerdo con estos resultados, los BT desarrollan una amplia variedad de cursos de acción, con unos circuitos altamente secuencializados y organizados para el logro de un resultado dado que cada acción ejecutada guarda una estrecha relación con la inmediatamente anterior. Se trata de circuitos de acción que implican un rico y variado contenido lo que, junto a las anteriores características, lleva a la obtención de una amplia y diversa tipología de resultados. Destaca además su capacidad para gestionar la propia actividad.

Los BD desarrollan una actividad más rígida y aislada, escasamente secuencializada, lo que origina un curso de acción corto y con escasas alternativas de acción, caracterizado además por un contenido lógico simple. Consecuentemente, este reducido curso de acción, conlleva una menor riqueza de resultados tanto en cantidad como en variedad y complejidad de contenido, más propio de períodos de desarrollo anteriores. Asímismo, los BD muestran poca iniciativa de acción, limitándose en algunas ocasiones a reproducir la propuesta de acción del adulto.

Por lo tanto, estos resultados permiten postular la existencia de diferencias en el proceso de elaboración de resultados de los bebés típicos y de los bebés con Síndrome de Down estudiados en esta temprana edad, en relación con el número, tipo y contenido de resultados obtenidos. 


\section{Discusıón}

Las diferencias halladas en el curso de la actividad dirigida hacia la elaboración de un resultado, constituyen índices de los cambios cognitivos que ocurren durante la infancia temprana en el proceso de organización e integración de la conducta (Jennings, 2004). Es por ello que la conducta dirigida hacia un logro constituye un aspecto importante en muchas teorías generales sobre el desarrollo (por ejemplo Bandura, 1997; Erikson, 1950), tratando de desvelar las distintas manifestaciones del curso de la acción que evidencian esos cambios cognitivos.

En este sentido, los resultados obtenidos en nuestro trabajo revelan cinco indicadores conductuales de las subyacentes diferencias cognitivas entre BT y BD en su proceso de elaboración de resultados: 1) la secuencialidad de la acción; 2) la flexibilidad/rigidez de la conducta; 3) el contenido lógico del curso de la acción; 4) la gestión de la acción y 5) las acciones co-ocurrentes a los resultados. Por lo tanto, estos resultados corroboran la hipótesis de partida relativa a la existencia de una mayor complejidad y flexibilidad en el curso de actividad entre los bebés típicos, complementariamente a los aportados en estudios previos similares (Sastre, 1996; Sastre et al., 2003).

Por otro lado, es importante destacar que estos índices reflejo de las diferencias cognitivas entre BT y BD en su proceso de obtención de resultados, son similares a los obtenidos por Jennings (2004) en su trabajo con niños típicos de $1 ; 3$ y $2 ; 11$ años de edad, reflejo en este caso, de los cambios cognitivos propios de la infancia.

Un aspecto importante en relación a las diferencias obtenidas, es el hecho de que en este proceso de elaboración de resultados y del saber, el niño no se encuentra solo. Desde la perspectiva neuroconstructivista defendida, el desarrollo cognitivo no es un suceso individual ni aislado. El bebé construye su propio desarrollo de la inteligencia en interacción con el medio físico y social que le rodea (Pastor y Sastre, 1994). Consecuentemente, es preciso atender asimismo al rol que juega el adulto que lo acompaña en esta situación social de elaboración de resultados.

La organización y complejidad de la actividad infantil, es el índice principal que permite al adulto ajustar sus propuestas a la capacidad y nivel de producción del bebé y obtener unos efectos optimizadores. En los bebés con Síndrome de Down, la pobre organización de la actividad dificulta la intervención adulta. El adulto carece de información para tutelar de forma óptima, respetando la finalidad perseguida y la zona de desarrollo próximo del bebé, por lo que sus propuestas son más desajustadas. En ocasiones, incluso puede llegar a convertirse en un factor distorsionante y paralizador de la actividad del niño, dada su excesiva intervención o sus propuestas no asimilables y cada vez más complejas, a las que el niño no puede responder (Sastre, 2001; Sastre y Pastor, 2001; Verba et Sastre, 2001). Es decir, la ganancia cognitiva reclama un feedback entre niño y adulto: el adulto necesita conocer unos índices que le permitan actuar de forma relacionada con el objetivo perseguido por el niño. A la vez, precisa que el niño responda a sus proposiciones con el fin de obtener nuevos índices orientadores de su actividad y comprobar el alcance y repercusión de sus informaciones. Al mismo tiempo, el niño requiere que el adulto le 
ofrezca posibilidades y/o interpretaciones a su acción, ajustadas a sus objetivos, capacidad y dificultades (Merino, 2003; Sastre, 2005).

Queda patente por tanto, la necesidad de atender a las dinámicas interactivas diferenciales que se establecen entre el niño y el adulto, dado que el desarrollo cognitivo no es un proceso aislado sino en interacción. Consecuentemente, se requieren pautas interactivas propias y adecuadas a las características diferenciales de cada bebé debidos a sus repercusiones en la construcción y gestión intelectual, con el fin de que a la insuficiencia biológica inicial se añada el menor número de interacciones sociales y educativas no adecuadas que pueden engrandecerla (Cambrodí, 1983). Durante los primeros años de vida, los factores biológicos tienden a fundirse con los factores socio-económicos y las condiciones de la interacción del niño con su entorno familiar, incidiendo conjuntamente en su desarrollo (de Kloet, Sibug, Helmerhorst \& Schmidt, 2005; Leckman, Feldman, Swain, Eicher, Thompson \& Mayes, 2004; Mayes, Swain \& Leckman, en prensa).

Cada vez es más patente la necesidad y posibilidad de una intervención temprana diferencial para la optimización del desarrollo de los sujetos con un funcionamiento cognitivo alternativo, que atienda globalmente sus particularidades, así como el papel del adulto para su optimización (Caughy, Huang, Miller \& Genevro, 2004; Figueras, 2004; Reynolds, 2004).

\section{Bibliografía}

ANDERSON, V., LEVIN, H. S. y JACOBS, R. (2002). Executive functions after frontal lobe injury: A developmental perspective. En D. T. Stuss y R. T. Knight (eds.), Principles of frontal lobe function (pp. 504-527). New York: Oxford University Press. ANGUERA, M. T. (1990). Metodología observacional. En J. Arnau, M. T. Anguera y J. Gómez (eds.), Metodología de la investigación en Ciencias del Comportamiento (pp. 125-236). Murcia: Secretariado de Publicaciones de la Universidad de Murcia.

BAKEMAN, R. y QUERA, V. (2002). Analyzing Interaction: Sequential Analysis with SDIS and GSEQ. New York: Cambridge University Press.

BANDURA, A. (1997). Self-efficacy: The exercise of control. New York: Freeman.

BENES, F. M., TAYLOR, J. B. y CUNNINGHAM, M. (2000). Convergence and plasticity of monoaminergic systems in the medial prefrontal cortex during postnatal period: Implications for the development of psychopathology. Cerebral Cortex, 10, 1014-1027.

BIDEAUD, J. (1988). Logique et bricolage chez l'enfant: classer et ordenner. Lille: Presses Universitaires de Lille.

BRUNER, J. S. (1984). Acción, pensamiento y lenguaje. Madrid: Alianza Editorial.

CAMBRODÍ, A. (1983). Principios de psicología evolutiva del deficiente mental. Barcelona: Herder.

CAMBRODÍ, A. y SASTRE, S. (1993). Escales d'Observació Sistemàtica 0-3 anys. Barcelona: P.P.U.

CAUGHY, M. O., HUANG, K., MILLER, T. y GENEVRO, J. L. (2004). The effects of the Healthy Steps for Young Children Program: results from observations of parenting and child development. Early Childhood Research Quarterly, 19, 611-630. 
CHRISTOFF, K. y GABRIELI, J. (2000). The frontopolar cortex and human cognition: evidence for a rostrocaudal hierarchical organization within the human prefrontal cortex. Psychobiology, 28, 168-186.

CSIBRA, G., BIRO, S., KOOS, O. y GERGELY, G. (2003). One-year-old infants use teleological representations of actions productively. Cognitive Science, 27, 111-133.

DE KLOET, E. R., SIBUG, R. M., HELMERHORST, F. M. y SCHMIDT, M. (2005). Stress, genes and the mechanism of programming the brain for later life. Neuroscience and Biobehavioral Reviews, 29, 271-281.

DIAMOND, A. (2001). Prefrontal cortex development and development of cognitive functions. En The International Encyclopedia of the Social and Behavioral Sciences, 4 (3), article: 198. London: Elsevier Press.

- (2002). Normal development of prefrontal cortex from birth to young adulthood: Cognitive functions, anatomy and biochemistry. En D. T. Stuss y R. T. Knighte (eds.). Principles of Frontal Lobe Function (pp. 466-503). New York: Oxford University Press.

ERIKSON, E. (1950). Childhood and society. New York: Norton.

ESCOLANO, E. (2003). Organización y contenido lógico de la acción en bebés típicos y bebés con Síndrome de Down (1;3 a.). Tesis de Licenciatura no publicada. Logroño: Universidad de La Rioja.

FIGUERAS ALOY, J. (2004). Coordinación interdisciplinaria de la atención al recién nacido de alto riesgo. Propuestas de actuación. Boletín de Pediatría, 44, 182-184.

HEYDER, K., SUCHAN, B. y DAUM, I. (2004). Cortico-subcortical contributions to executive control. Acta Psychologica, 115, 271-289.

HOMMEL, B. (2003). Planning and representing Intencional Action. TheScientificWorldJOURNAL, 3, 593-608.

JENNINGS, K. D. (2004). Development of goal-directed behaviour and related selfprocesses in toddlers. International Journal of Behavioral Development, 28 (4), 319-327.

KRIPPENDORF, K. (1980). Content analysis: An introduction to its methodology. Beverly Hills: Sage.

LANGER, J. (1980). The origins of Logic: Six to twelve months. New York: Academic Press.

- (1982). From prerepresentational to representational cognition. En G. Forman (ed.), Action and thought. Nueva York: Academic Press.

- (1986). The origins of Logic: One to two years. Orlando: Academic Press.

- (1990). Early cognitive development: Basic funtions. En C. A. Hauert (ed.), Developmental Psychology: Cognitive, perceptuo-motor and neuro-psychological perspectives, 19-42. Amsterdam: North Holland.

- (2000). The heterochronic evolution of primates. Cognitive development. En S.T. Parker, J. Langer y L. Mckinney (eds.), Biology, Brains and Behavior (pp. 215236). Oxford: James Currey Ltd.

LECKMAN, F., FELDMAN, R., SWAIN, J. E., EICHER, V., THOMPSON, N. y MAYES, L.C. (2004). Primary parental preoccupation: circuits, genes, and the crucial role of the environment. Journal of Neural Transmision, 111, 753-771. 
MANDLER, J. (1998). Representation. En W. Damon y R. Lerner (eds.), Handbook of Child Psychology. Volume II: Cognition, Preception and Language (5th Edition) (pp. 268-273). New York: Wiley \& Sons.

MARESCHAL, D. y SHULTZ, T.R. (1997). From neural constructivism to children's cognitive development. Bridgimg the gap. Behavioral and Brain Sciences, 20 (4), 571.

MAYES, L. C., SWAIN, J. E. y LECKMAN, J. F. (en prensa). Parental attachment systems: neural circuits, genes, and experiential contributions to parental engagement. Clinical Neuroscience Research.

MERINO, N. (2003). Interacción e interferencia: una perspectiva diferencial y evolutiva (1,3 a.-1;9 a.). Tesis de Licenciatura no publicada. Logroño: Universidad de La Rioja.

MOUNOUD, P. (1983). L'évolution des conduites de prehension comme illustration d'un modele du développement. En S. Schonen (ed.), Le développement dans la première anneé (pp. 75-106). Paris: PUF.

PASTOR, E. y SASTRE, S. (1994). Desarrollo de la inteligencia. En V. Bermejo (ed.), Desarrollo cognitivo, 191-213. Madrid: Síntesis.

PIAGET, J. (1976). "Le possible, I'impossible et le nécessaire: Les recherches en cours ou projetées au Centre international d'épistémologie génétique". Archives de psychologie, 44 (172), 281 -299. Trad castellano: "Lo posible, lo imposible y lo necesario". Monografías de Infancia y Aprendizaje, 2 (número especial de homenaje a Piaget), 1981, 108-121.

- (1983). Piaget's theory. In W. Kessen (Comp.), En Handbook of Child Psychology, 1, 294-356. New York: Wiley.

POCH, M. L., SASTRE, S., MERINO, N., ESCOLANO, E., FARRIOL, R. y GIL, J. (2003). Unidades de seguimiento del desarrollo. Revista de Atención Temprana, $6(1), 23-27$.

QUARTZ, S. R. y SEJNOWSKI, T. J. (1994). Beyond modularity: Neural evidence for constructivst principles in development. Behavioral and Brain Sciences, 17, 25-26.

- (1997). The neural basis of cognitive development: A constructivist manifest. Behavioural and Brain Sciences, 20, 537-596.

REYNOLDS, A. J. (2004). Research on early childhood interventions in the confirmatory mode. Children and Youth Services Review, 26, 15-38.

SASTRE, S. (1996). Estudios de desarrollo cognitivo precoz: alcance, aplicaciones y límites en el proceso de deficienciación. Universitas Tarraconenses (Revista de Psicología), 18 (2), 77-107.

- (2001). Desarrollo cognitivo diferencial e intervención psicoeducativa. Contextos Educativos, 4, 95-117.

- (2005). Tutoring Adjustment And Infants' Cognitive Gain. En L. Anolli, S. Duncan Jr, M.S. Magnusson y G. Riva (eds.), The Hidden Structure of Interaction: From Neurons to Culture Patterns (pp. 195-206). Amsterdam: IOS Press.

SASTRE, S., BRETÓN, P., ESCORZA, J., LÁZARO, V., PASCUAL, M. T., POCH, M. L., SOARES, Y. y MERINO, N. (2000). Desarrollo cognitivo e inhibición, prevención e intervención en La Rioja. Memoria de investigación. Consejería de Salud de la Comunidad Autónoma de La Rioja. 
SASTRE, S., BRETÓN, P., ESCORZA, J., LOZANO, R., PASCUAL, M. T., POCH, M. L., RUIZ, Y., SOARES, Y., MERINO, N. y ESCOLANO, E. (2003). Cognición Temprana Diferencial y Función Ejecutiva. Memoria de investigación. Consejería de Salud de la Comunidad Autónoma de La Rioja.

SASTRE, S., ESCOLANO, E. y MERINO, N. (2004). Observación sistemática de la cognición temprana: funciones ejecutivas, lógica e interacción. Metodología de las Ciencias del Comportamiento, volumen especial, 557-566.

SASTRE, S. y PASTOR, E. (2001): Modalidades de tutela de "gestión cognitiva" en bebés trisómicos. Infancia y Aprendizaje, 93, 35-52.

SOMMERVILLE, J. A. y WOORDWARD, A. L. (2005). Pulling out the intentional structure of action: The relation between action processing and action production in infancy. Cognition, 95, 1-30.

STUSS, D. T. y KNIGHT, R. T. (2002). Introduction. En D. T. Stuss y R. Knight (eds.), Principles of Frontal Lobe Function, 1-7. New York: Oxford University Press.

VARGAS, J. D. (1999). Modalidades sociocognitivas de construcción del saber en la interacción entre iguales en las primeras edades. Tesis Doctoral inédita. Universitat Rovira i Virgili, Tarragona.

VERBA, M. y SASTRE, S. (2001). Les interactions de tutelle avec les bébes normaux et les enfants trisomiques: I'apport de I'enfant dans I'ajustament de l'adulte. Enfance, 2, 197-214.

VILLARES, L. (1999). Modalidades de tutela paterna y efectos en el desarrollo cognitivo del hijo (1;0 - 2;0 a.) Tesis Doctoral inédita. Universidad Rovira i Virgili, Tarragona.

WOODWARD, A. L. (1998). Infants selectively encode the goal object of an actor's reach. Cognition, 69, 1-34.

WOODWARD, A. L. y GUAJARDO, J. J. (2002). Infants' understanding of the point gesture as an object-directed action. Cognitive Development, 83, 1-24.

WOODWARD, A. L. y SOMMERVILLE, J. A. (2000). Twelve-month-old infants interpret action in context. Psychological Science, 11, 73-77.

WOODWARD, A. L., SOMMERVILLE, J. A. y GUAJARDO, J. J. (2001). How infants make sense of intentional action. En B. F. Malle, L. J. Moses y D. A. Baldwin (eds.), Intentions and intentionally: Foundations of social cognition (pp. 149169). Cambridge, MA: MIT Press.

ZACKS, J. M. y TVERSKY, B. (2001). Event structure in perception and conception. Psychological Bulletin, 127 (1), 3-21.

ZELAZO, P. H., CRAIK, F. M. I. y BOOTH, L. (2004). Executive function across the life span. Acta Psychologica, 115, 167-183. 